\title{
1D-Quasiperiodic Operators. Latent Symmetries
}

\author{
V. A. Mandelshtam and S. Ya. Zhitomirskaya \\ Academy of Sciences of the USSR, International Institute of Earthquake Prediction Theory and \\ Mathematical Geophysics ${ }^{\star}$, Warshavskoye sh., 79, k.2, SU-119556 Moscow, USSR
}

Received December 15, 1990

\begin{abstract}
A large class of discrete quasiperiodic operators is shown to be decomposed into orbits of $S L(2, \mathbb{Z})$ action with equal densities of states. Moreover under some natural assumptions all nontrivial representatives of the mentioned action transform operators with pure point spectrum into those with absolutely continuous spectrum. Some applications of these results are presented.
\end{abstract}

\section{Introduction}

Let us consider the general family of quasiperiodic operators acting in $l^{2}(\mathbb{Z})$ through the formula

$$
(H \Psi)_{l}=\sum_{j=-\infty}^{\infty} \Psi_{l-j} f_{j}(\Phi+l \alpha), \quad f_{j} \in C\left(S^{1}\right), \alpha \in \mathbb{R} \backslash \mathbb{Q} .
$$

This paper is an attempt of outlook on this class as a whole in order to reveal which almost periodic features are responsible for various spectral properties and thus to provide some old results with a new understanding.

During the 80 's there was a great amount of interest in almost periodic operators. The balance between generality and concreteness was periodically driven to either side. Perhaps the most attention was paid to investigations of the almost-Mathieu operator

$$
(H \Psi)_{l}=\Psi_{l+1}+\Psi_{l-1}+\lambda \cos (\alpha l+\Phi) \Psi_{l}
$$

and related models.

The almost-Mathieu equation arose from the model of Bloch electron in the uniform magnetic field [1] and has been studied extensively both from physical and mathematical points. It seemed to have very interesting properties but (in comparison with a related $2 D$-operator) a simple easy to handle form.

* Telex 411628 MITPA SU; Fax (095) 3107032 
The rigorous results concerning almost-Mathieu operator can be divided into two classes. Those which hold for more general operator families [2-5] etc.), and those which use essentially the almost-Mathieu specificity [6-10]. The theorems of the first class concern usually the nicer spectral properties but cover mainly the considerably smaller sets of parameters than the results of the second one. The specificity used is the symmetry of the almost-Mathieu family with respect to the Fourier transform (the "Aubry duality") which gives rise to proving the positivity or vanishing of Lyapunov exponents $\gamma(E, \lambda)$ for corresponding values of $\lambda$. This implies that the spectral measure does not contain an absolutely-continuous component for $\lambda>2$ and there are no pure point spectrum for $\lambda<2$. We will call such results as theorems "up to" the singular-continuous spectrum because clearing up whether there exists a singular-continuous spectrum for $\lambda \neq 2$ and the proof of the believable pure singular-continuity of the spectral measure for $\lambda=2$ will give a complete description of the spectrum.

The duality argument at first appeared in [6] as a quasitheorem and was proved rigorously in [7] and [8] by different methods. It has been uncertain what is the nature of the duality and whether this argument can be extended to the wider classes of almost-periodic problems.

In this paper we will show that symmetry properties of the whole class of $1 D$ almost-periodic operators are caused by the existence of some gauge group for related $2 D$ Hamiltonians. Thus a physically intuitive connection between almostperiodic and $2 D$ operators in a uniform magnetic field becomes a mathematical fact which enables one to find a group of transforms (including the Fourier transform) that preserves the integrated density of states. These are the results of Theorems 1 and 2. Theorem 3 shows that under some natural assumptions every orbit of the above mentioned group action includes at most one isospectral class of operators with a pure point spectrum, all other operators in the orbit possessing pure absolutely-continuous spectrum. Theorem 4 is an example of application of Theorem 3. Theorem 5 relying on Theorem 2 describes the spectral properties up to the singular-continuous spectrum of some operator family.

Thus the essence of our method is that deriving $1 D$ quasiperiodic operator from the $2 D$ Hamiltonian one should not forget the initial problem. We remark that all known proofs of the Aubry duality have in fact the two-dimensional nature. All the latent symmetries become explicit when regarding the related $2 D$ operator.

Let us give now the precise formulations.

We consider an operator $\mathbf{H}$ of the form (1). It is connected with the matrix of the Fourier coefficients $B=B(k, j)=\left\|b_{k, j}\right\|$ appearing in the expansion of the functions

$$
f_{j}(x)=\sum_{j=-\infty}^{\infty} b_{k, j} \exp (i k(x-j \alpha / 2)) .
$$

We will use the notation $\mathbf{H}_{B, \alpha}^{\Phi}$ for operator (1). (The indices $\alpha, \Phi$ would be ignored in all objects connected with $\mathbf{H}_{B}$ if it would not lead to ambiquity; in order to avoid rather cumbersome notations we will sometimes use $b_{l}$ for $b_{k, j}$, where $\left.l=(k, j) \in \mathbb{Z}^{2}\right)$. Sometimes it will be convenient to deal with a diagram corresponding to the matrix $B$, which can be built by omitting all zero coefficients $b_{k, j}$ and providing all nonzero $b_{k, j}$ with vectors going from the point $(0,0)$ to the point $(k, j)$. Denote by $\Sigma$ the set of operators $\mathbf{H}_{B}$ such that $b_{l}=\overline{b_{-l}}$ and $b_{l} \in l^{1}\left(\mathbb{Z}^{2}\right)$. Let $\mathbf{H}_{B}(\Lambda)$ be the operator $\mathbf{H}_{B}$ restricted to $l^{2}(-\Lambda, \Lambda) \subset l^{2}(\mathbb{Z})$. Denote, as usual, by $(2 \Lambda+1) \mathbf{N}_{B}(\lambda ; \Lambda)$ the number of eigenvalues $\lambda_{i}(\Lambda)$ of $\mathbf{H}_{B}(\Lambda)$ which are less than $\lambda$, i.e. $\lambda_{i}(\Lambda) \leqq \lambda$. 
Take $\mathbf{H}_{B} \in \Sigma$. It follows from the general facts for the random metrically transitive operators [11] that

1) $\mathbf{H}_{B}$ is an essentially selfadjoint operator defined on the space of finite sequences.

2) There is a deterministic ( $\Phi$-independent) measure $d \mathbf{N}_{B}(\lambda)$ which is the weak $\Lambda \rightarrow \infty$ limit of the sequence $d \mathbf{N}_{B}(\lambda ; \Lambda)$.

3) Let $d \mathbf{E}_{B}^{\Phi}(\lambda)$ be the identity resolution of $\mathbf{H}_{B}^{\Phi}$. Then

$$
d \mathbf{N}_{B}(\lambda)=\int_{S^{1}}\left(d E_{B}^{\Phi}(\lambda) e_{0}, e_{0}\right) d \Phi
$$

where the vector $e_{0} \in l^{2}, e_{0}(n)=\delta(0, n)$.

\section{The Connection Between the Quasiperiodic Operators and $2 D$ Hamiltonians in the Uniform Magnetic Field}

Given $\mathbf{H}_{B} \in \Sigma$ let us consider the Hamiltonian $\mathbf{L}_{B}$ acting in $l^{2}\left(\mathbb{Z}^{2}\right)$ :

$$
\left(L_{B} \Psi\right)_{k}=\sum_{l \in \mathbb{Z}^{2}}^{\infty} \Psi_{k-l} b_{l} \exp \left(i \alpha \Theta_{k, k-l}\right), \quad k=\left(k^{1}, k^{2}\right), l=\left(l^{1}, l^{2}\right) \in \mathbb{Z}^{2}
$$

where the gauge field $\alpha \Theta_{k, l}$ corresponds to the uniform magnetic field with the flux $\alpha$ through the unit cell. Immerse the lattice $\mathbb{Z}^{2}$ into $\mathbb{R}^{2}$. Let $x, y$ be the coordinates in $\mathbb{R}^{2}$. Given $k, l \in \mathbb{Z}^{2}$ consider the vector $t_{k, l} \in T_{k} \mathbb{R}^{2}$ with the coordinates $l^{1}-k^{1}$, $l^{2}-k^{2}$. Here $T_{k} \mathbb{R}^{2}$ is the tangent space identified with $\mathbb{R}^{2}$. Fix $\Theta \in \Lambda^{1} \mathbb{R}^{2}$ being the differential 1-form such that $d \Theta=d x \wedge d y$. The form $\Theta$ is defined, uniquely, up to the gradient of any function $f: \mathbb{R}^{2} \rightarrow \mathbb{R}$. Now we can choose $\Theta_{k, l}$ being equal to $\Theta\left(t_{k, l}\right)$. Note that all "physical" values connected with $\mathbf{L}_{B}$ are gauge invariant, i.e. independent on the concrete choice of the gauge field $\Theta_{k, l}=\Theta\left(t_{k, l}\right)$ such that $d \Theta=d x \wedge d y$. The wave functions $\Psi(x, y)$ of operator $\mathbf{L}_{B}^{\Phi+\operatorname{grad} f(x, y)}$ are exactly those of operator $\mathbf{L}_{B}^{\Theta}$ multiplied by the phase factor $\exp \{i f(x, y)\}$. It turns out that the group of transformations of the Hamiltonian $\mathbf{L}_{B}$ due to the gauge arbitrariness leads to the decomposition of the set of quasiperiodic operators into orbits consisting of operators with equal densities of states (Theorem 2). This fact is a generalization of the Aubry duality which follows within our method from the symmetry of the lattice with respect to the rotations by the angle $\pi / 2$.

Consider the ensemble $Y$ of the lattice paths $\gamma=\left(\gamma_{0}, \ldots, \gamma_{t}\right), \gamma_{j}=\left(\gamma_{j}^{1}, \gamma_{j}^{2}\right) \in \mathbb{Z}^{2}$, $\gamma_{0}=\gamma_{t}=0, t=|\gamma|$. Here $t=|\gamma|$ is the length of the path $\gamma$. Let

$$
p_{B, \alpha ; \lambda}(\gamma)=\left(\sum_{j=1}^{t} b_{\gamma_{j}-\gamma_{j}-1}\right) \exp \{i \alpha S(\gamma)\} \frac{\lambda^{|\gamma|}}{|\gamma| !}
$$

be the statistical weight of the path $\gamma$. Denote by $\Xi_{B, \alpha ; \lambda}$ the partition function over the ensemble $Y$. It is straightforward that $\Xi_{B, \alpha ; \lambda}=\left(\exp \left\{\lambda \mathbf{L}_{B}\right\} e_{j}, e_{j}\right)$ for any $j=\left(j^{1}, j^{2}\right) \in \mathbb{Z}^{2}$. Here $e_{j} \in l^{2}\left(\mathbb{Z}^{2}\right), e_{j}(n, m)=\delta\left(n, j^{1}\right) \delta\left(m, j^{2}\right)$.

It turns out that $\Xi_{B, \alpha ; \lambda}$ can be represented in terms of the operator $\mathbf{H}_{B}$.

Theorem 1. For every irrational $\alpha$ and $\mathbf{H}_{B} \in \Sigma$,

$$
\int \exp \{-\lambda t\} d \mathbf{N}_{B, \alpha}(t)=\Xi_{B, \alpha ; \lambda} .
$$

Proof. Consider the area functional $S(\gamma)$ determined on all (not necessarily closed) lattice paths: $S(\gamma)=\int_{\tilde{\gamma}} \Theta, \Theta \in \Lambda^{1} \mathbb{R}^{2}, d \Theta=d x \wedge d y, \tilde{\gamma} \subset \mathbb{R}^{2}$ is the image of the path $\gamma$ on $\mathbb{Z}^{2}$ after the natural immersion of $\mathbb{Z}^{2}$ into $\mathbb{R}^{2}$. Fix $\Theta=y d x$. It is obvious that the 
concrete choice of the functional $S(\gamma)$ is equivalent to the choice of the gauge field; it will be convenient to consider area formulas instead of gauges.

Fix $k \in \mathbb{N} \cup\{0\}, z_{1}, z_{2} \in S^{1}=\{|z|=1\} \subset \mathbb{C}$. Consider the ensemble $\Pi^{k}$ of the lattice paths $\gamma=\left(\gamma_{0}, \ldots, \gamma_{k}\right), \gamma_{j}=\left(\gamma_{j}^{1}, \gamma_{j}^{2}\right) \in \mathbb{Z}^{2}, \gamma_{0}=0$ with the fixed length $k$. Let the statistical weight $p_{B, \alpha ; z_{1}, z_{2}}(\gamma), \gamma \in \Pi^{k}$, be equal to

$$
\left(\prod_{j=1}^{k} b_{\gamma_{j}-\gamma_{j-1}}\right) \exp \{i \alpha S(\gamma)\} z_{1}^{\gamma_{k}^{1}} z_{2}^{\gamma_{k}^{2}}, \quad k \geqq 0 .
$$

Denote by $\mathbf{P}_{B, \alpha}^{k}\left(z_{1}, z_{2}\right)$ the corresponding partition function. Consider the function $\varphi\left(z_{1}, z_{2}\right)=\sum\left|b_{k, l}\right| z_{1}^{k} z_{2}^{l}$. It follows from the definition of $\sum$ that $\varphi\left(z_{1}, z_{2}\right) \in C\left(S^{1} \times S^{1}\right)$, and one can apply the Cauchy theorem to it. Taking into account the estimate

$$
\left|\mathbf{P}_{B, \alpha}^{k}\left(z_{1}, z_{2}\right)\right| \leqq \varphi^{k}(1,1),
$$

we obtain that the same property of the function $\mathbf{P}_{B, \alpha}^{k}\left(z_{1}, z_{2}\right)$ holds. Thus if $\mathbf{P}_{B, \alpha}^{k}\left(z_{1}, z_{2}\right)=\sum_{j_{1}, j_{2}} C_{B, \alpha ; j_{1}, j_{2}}^{k} z_{1}^{j_{1}} z_{2}^{j_{2}}$, then

$$
C_{B, \alpha ; 0,0}^{k}=\iint \mathbf{P}_{B, \alpha}^{k}\left(\exp \left\{i \Phi_{1}\right\}, \exp \left\{i \Phi_{2}\right\}\right) d \Phi_{1} d \Phi_{2} .
$$

Let us introduce the "inverse temperature" $\lambda, \lambda \in \mathbb{C}$. Let $\Pi$ be the disjunct union of ensembles $\Pi^{k}$. Put the statistical weight $p_{B, \alpha ; z_{1}, z_{2} ; \lambda}(\gamma), \gamma \in \Pi^{k} \subset \Pi$ equal to $\frac{\lambda^{k}}{k !} p_{B, \alpha ; z_{1}, z_{2}}(\gamma)$. The partition function $\mathbf{P}_{\boldsymbol{B}, \alpha}\left(z_{1}, z_{2} ; \lambda\right)$ over the ensemble $\Pi$ is of course equal to $\sum \mathbf{P}_{B, \alpha}^{k}\left(z_{1}, z_{2}\right) \frac{\lambda^{k}}{k !}$. It follows from estimate (5) that for any $\lambda \in \mathbb{C}$ the
value

$$
\left|\mathbf{P}_{B, \alpha}\left(z_{1}, z_{2} ; \lambda\right)\right| \leqq \exp \{|\lambda| \varphi(1,1)\},
$$

therefore $\mathbf{P}_{\boldsymbol{B}, \alpha}\left(z_{1}, z_{2} ; \lambda\right)$ is an entire function of $\lambda$, for $\left|z_{1}\right|=\left|z_{2}\right|=1$. There exists a natural family of recurrent equations on the partition functions $\mathbf{P}_{B, \alpha}^{k}\left(z_{1}, z_{2}\right)$. In fact given $\gamma^{(k)}=\left(\gamma_{0}, \ldots, \gamma_{k}\right), \gamma^{(k+1)}=\left(\gamma^{(k)}, \gamma_{k+1}\right)$ such that $\gamma_{k+1}-\gamma_{k}=(j, l)$ we have

$$
S\left(\gamma^{(k+1)}\right)=S\left(\gamma^{(k)}\right)+j l / 2+\gamma_{k}^{2} j,
$$

hence

$$
\begin{aligned}
\mathbf{P}_{B, \alpha}^{k+1}\left(z_{1}, z_{2}\right) & =\sum b_{j, l} \mathbf{P}_{B, \alpha}^{k}\left(z_{1}, z_{2} \exp (i \alpha j)\right) z_{1}^{j} z_{2}^{l} \exp (i \alpha j l / 2), \quad k \geqq 0, \\
\mathbf{P}_{B, \alpha}^{0}\left(z_{1}, z_{2}\right) & =1 .
\end{aligned}
$$

System (6) is equivalent to the functional-differential equation for $\mathbf{P}_{\boldsymbol{B}, \alpha}\left(z_{1}, z_{2} ; \lambda\right)$ :

$$
\begin{gathered}
\frac{d \mathbf{P}}{d \lambda}=\sum b_{j, l} \mathbf{P}\left(z_{1}, z_{2} \exp (i \alpha j), \lambda\right) z_{1}^{j} z_{2}^{l} \exp (i \alpha l j / 2) \\
\mathbf{P}\left(z_{1}, z_{2}, 0\right)=1 .
\end{gathered}
$$

Define $\mathbf{Q}_{B, \alpha}^{\Phi}(\lambda) \in l^{1}, \Phi \in S^{1}, \lambda \in \mathbb{C}$ as a vector with the coordinates

$$
\left(\mathbf{Q}_{B, \alpha}^{\Phi}(\lambda)\right)_{l}=\int_{0}^{2 \pi} \mathbf{P}_{B, \alpha}\left(e^{i \Phi}, e^{i \delta}, \lambda\right) \exp (-i l \delta) d \delta
$$

Note that $\mathbf{Q}_{B, \alpha}^{\Phi}(\lambda)$ belongs to $l^{1}$ because it is a sequence of Fourier coefficients of a continuous function. For every $l \in \mathbb{Z}$ the function $\left(\mathbf{Q}_{B, \alpha}^{\Phi}\right)_{l}$ depends analytically on $\lambda$. 
Equation (7) can be rewritten in terms of $\mathbf{Q}_{B, \alpha}^{\Phi}$ as a Cauchy problem

$$
\begin{aligned}
\frac{d \mathbf{Q}_{B, \alpha}^{\Phi}(\lambda)}{d \lambda} & =\mathbf{H}_{B, \alpha}^{\Phi} \mathbf{Q}_{B, \alpha}^{\Phi}(\lambda), \\
\mathbf{Q}_{B, \alpha}^{\Phi}(0) & =\mathbf{Q}^{(0)},
\end{aligned}
$$

where $\mathbf{Q}^{(0)} \in l^{1}, \mathbf{Q}^{(0)}(n)=\delta(n, 0)$.

Note that for every $k \geqq 0$ the vector $\left(\mathbf{H}_{B, \alpha}^{\Phi}\right)^{k} \mathbf{Q}^{(0)}$ is well defined and belongs to $l^{1}$, because of the estimate

$$
\sum_{l \in \mathbb{Z}}\left|\left(\left(\mathbf{H}_{B, \alpha}^{\Phi}\right)^{k} \mathbf{Q}^{(0)}\right)_{l}\right| \leqq \sum_{j_{i}, m_{i} \in \mathbb{Z}}\left|b_{j_{1}, m_{1}}\right| \ldots\left|b_{j_{k}, m_{k}}\right|=\varphi^{k}(1,1) .
$$

Since $\mathbf{Q}_{B, \alpha}^{\Phi}(\lambda)$ is an analytical solution of the Cauchy problem (8), hence it equals $\exp \left(\lambda \mathbf{H}_{B, \alpha}^{\Phi}\right) \mathbf{Q}^{(0)}$. Taking into account that

$$
\Xi_{B, \alpha ; \lambda}=\int_{0}^{2 \pi}\left\{\mathbf{Q}_{B, \alpha}^{\Phi}(\lambda)\right\}_{0} d \Phi
$$

we obtain

$$
\begin{aligned}
\Xi_{B, \alpha ; \lambda} & =\int_{0}^{2 \pi}\left(\exp \left(\lambda \mathbf{H}_{B, \alpha}^{\Phi}\right) \mathbf{Q}^{(0)}, \mathbf{Q}^{(0)}\right) d \Phi \\
& =\int_{0}^{2 \pi} \int_{-\infty}^{\infty}\left(\exp (\lambda t) d E_{B, \alpha}^{\Phi}(t) \mathbf{Q}^{(0)}, \mathbf{Q}^{(0)}\right) d \Phi=\int_{-\infty}^{\infty}\left(\exp (\lambda t) d \mathbf{N}_{B, \alpha}(t) .\right.
\end{aligned}
$$

Here we exploited formula (3). To prove the legality of changing the order of integration one should approximate $\exp (\lambda t)$ by a sequence of step functions $f_{k}(t)$ such that $f_{k}(t) \leqq \exp (\lambda t)$ and apply the Lebesgue and Levi theorems. Q.E.D.

\section{The "Duality"}

Because of the gauge invariance of the right-hand side of (4) it follows that the measure $d \mathbf{N}_{B, \alpha}(t)$ is invariant with respect to any area-preserving lattice transform. Moreover, given homomorphism

$$
A=\left(\begin{array}{ll}
a & b \\
c & d
\end{array}\right) \in G L(2, \mathbb{Z}),
$$

let $A(i, j) \in \mathbb{Z}^{2}$ be $(a i+b j, c i+d j)$. Define the induced action of $A$ on the matrices $B$ :

$$
\widehat{A} B(i, j)=B\left(A^{-1}(i, j)\right) .
$$

Theorem 2. Let $A \in G L(2, \mathbb{Z}), \mathbf{H}_{B} \in \Sigma, \alpha \notin \mathbb{Q}$, then

$$
\mathbf{N}_{\hat{A} B, \alpha}(E)=\mathbf{N}_{B, \alpha \operatorname{det} A}(E) \text {. }
$$

Proof. Given path $\gamma=\left(\gamma_{0}, \ldots, \gamma_{t}\right), \gamma_{i} \in \mathbb{Z}^{2}, \gamma_{0}=\gamma_{t}=0$, consider the corresponding path $\hat{\gamma}=\left(A \gamma_{0}, \ldots, A \gamma_{t}\right)$. Then

$$
\begin{aligned}
p_{\hat{A} B, \alpha ; \lambda}(\hat{\gamma}) & =\left(\prod_{j=1}^{t} b_{A^{-1}\left(\hat{\gamma}_{j}-\hat{\gamma}_{j-1}\right)}\right) \exp \{i \alpha S(\hat{\gamma})\} \frac{\lambda^{|\hat{\gamma}|}}{|\hat{\gamma}| !} \\
& =\left(\prod_{j=1}^{t} b_{\gamma_{j}-\gamma_{j-1}}\right) \exp \{i \alpha S(\gamma) / \operatorname{det} A\} \frac{\lambda^{|\gamma|}}{|\gamma| !}=p_{B, \alpha \operatorname{det} A, \lambda}(\gamma)
\end{aligned}
$$


Changing the summation over the paths $\gamma$ to the summation over $\tilde{\gamma}$ we obtain equality $\Xi_{\hat{A} B, \alpha ; \lambda}=\Xi_{B, \alpha \operatorname{det} A ; \lambda}$ which immediately gives the statement of the theorem.

In particular, if $A \in S L(2, \mathbb{Z})$, then

$$
\mathbf{N}_{\hat{A} B}(E)=\mathbf{N}_{B}(E) .
$$
The equality (11) turns into Aubry duality when $A$ is $\left(\begin{array}{rr}0 & 1 \\ -1 & 0\end{array}\right)$ and the diagram
corresponding to $\mathbf{H}_{B}$ is

Fig. 1

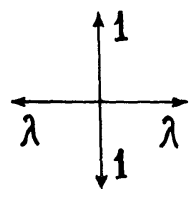

The generalization of the Aubry duality proven by Pastur [12] corresponds $A=\left(\begin{array}{rr}0 & 1 \\ -1 & 0\end{array}\right)$ and

$$
b_{i, j}=\varphi_{1}(j) \delta(0, i)+\varphi_{2}(i) \delta(0, j) .
$$

Theorem 2 enables to obtain results on spectral properties "up to" the singularcontinuous spectrum for different operator families invariant with respect to an arbitrary automorphism (see Sect. 4 for some examples).

\section{4. $S L(2, \mathbb{Z})$-Action}

Here we want to describe the families of $1 D$ quasiperiodic operators (1) with identical density of states, which are generated by the gauge transformations of $2 D$ Hamiltonians.

Unitary action of $S L(2, \mathbb{Z})$ in $L_{2}\left(S^{1} \times \mathbb{Z}\right)$ can be defined as follows. Let $A=\left(\begin{array}{rr}0 & 1 \\ -1 & 0\end{array}\right)$

\section{Set}

$\left(\mathbf{U}_{A} \varphi\right)(\beta, p)=\int_{0}^{2 \pi} d \Phi \sum_{n=-\infty}^{\infty} \exp (i \Phi p+i n(\beta+p \alpha)) \varphi(\Phi, n), \quad \Phi, \beta \in S^{1} ; p, n \in \mathbb{Z}-$ (the "Fourier transform").

$$
\text { For } A=\left(\begin{array}{ll}
1 & k \\
0 & 1
\end{array}\right) \text { set }
$$

$$
\left(\mathbf{U}_{A} \varphi\right)(\beta, p)=\exp (i p k(p \alpha / 2+\beta)) \varphi(\beta, p) .
$$

Proposition 1. The formulas (12)-(13) determine the

$$
S L(2, \mathbb{Z}) \text {-action on } L_{2}\left(S^{1} \times \mathbb{Z}\right) \text {. }
$$

Proof. Set $\mathbf{U}_{A B}=\mathbf{U}_{A} \mathbf{U}_{B}$. All we need is to decompose the every automorphism $A \in S L(2, \mathbb{Z})$ into a product of automorphisms of two above types. The correspond- 
ing fact is the discrete analog of the polar decomposition theorem: for every $A=\left(\begin{array}{ll}a & b \\ c & d\end{array}\right) \in S L(2, \mathbb{Z})$

$$
A=\left(\begin{array}{cc}
1 & k_{0} \\
0 & 1
\end{array}\right)\left(\begin{array}{rr}
0 & -1 \\
1 & 0
\end{array}\right)\left(\begin{array}{rr}
1 & k_{1} \\
0 & 1
\end{array}\right)\left(\begin{array}{rr}
0 & -1 \\
1 & 0
\end{array}\right) \cdots\left(\begin{array}{cc}
1 & k_{r} \\
0 & 1
\end{array}\right),
$$

where $k_{1}, \ldots, k_{r}$ are defined by

$$
\frac{c}{d}=\left[k_{r}, \ldots, k_{1}\right]=\frac{1}{k_{r}-\frac{1}{k_{r-1}-\ldots-\frac{1}{k_{1}}}} .
$$

Proposition 2. The following equality holds

$$
\mathbf{U}_{A} \mathbf{H}_{B} \mathbf{U}_{A}^{-1}=\mathbf{H}_{\hat{A} B} \text {. }
$$

Proof. It follows from the above that one needs to prove (16) only for the operators $\mathbf{U}_{A}$ of form (12), (13). Let $A$ be $\left(\begin{array}{ll}1 & k \\ 0 & 1\end{array}\right)$, i.e. $(\hat{A} B)(i, j)=b_{i-k j, j}$. Thus

$$
\left(\mathbf{H}_{\hat{A} B} \Psi\right)_{l}=\sum_{j=-\infty}^{\infty} \Psi_{l+j} \varphi_{j}(\Phi+l \alpha) \exp \{-i k j(\Phi+l \alpha+j \alpha / 2)\}
$$

hence

$$
\left(\mathbf{H}_{\hat{A} B} \mathbf{U}_{A} \Psi\right)_{l}=\sum_{j=-\infty}^{\infty} \Psi_{l+j} \varphi_{j}(\Phi+l \alpha) \exp (i l k(l \alpha / 2+\Phi))=\left(\mathbf{U}_{A} \mathbf{H}_{B} \Psi\right)_{l}
$$

The proof for case (12) is similar to this one.

Note that the group $\mathbf{G}=\left\{\left(\begin{array}{ll}1 & k \\ 0 & 1\end{array}\right)\right\}_{k \in \mathbb{Z}} C S L(2, \mathbb{Z})$ acts in $L_{2}\left(S^{1} \times \mathbb{Z}\right)$ as a group of operators which are unitary in $l^{2}$ for every fixed $\Phi \in S^{1}$. Thus $\mathbf{G}$ preserves the spectral properties of $\mathbf{H}_{B}$. However the action of automorphisms $A \in S L(2, \mathbb{Z}) \backslash \mathbf{G}$ changes essentially the spectra of $\mathbf{H}_{B}$ though it preserves the density of states. The precise assertion is given in Theorem 3. We need the following definition.

Consider the ergodic dynamical system $\left(M, \mu, T_{x}\right), x \in \mathbb{Z}^{d}$. The operator family $\mathbf{L}_{\Phi}: l^{2}\left(\mathbb{Z}^{d}\right) \rightarrow l^{2}\left(\mathbb{Z}^{d}\right), \Phi \in M$ will be called the metrically transitive (mt) if $R_{x} L_{\Phi} R_{x}^{-1}$ $=\mathbf{L}_{T_{x} \Phi}, \Phi \in M$, where $R_{x}$ is a lattice shift to the vector $x:\left(R_{x} \varphi\right)(n)=\varphi(n+x), n \in \mathbb{Z}^{d}$.

We will say that mt-operator $\mathbf{L}_{\Phi}$ possess $\mathbf{S}$-spectrum if the following three conditions hold:

1) The spectrum of $\mathbf{L}_{\Phi}$ is pure point for a.e. $\Phi \in M$.

2) The integrated density of states $\mathbf{N}_{L}(E)$ is absolutely continuous.

3) There exist measurable multi- (but a.e. finitely) valued functions

$$
\Lambda(\Phi)=\left(\Lambda^{1}(\Phi), \ldots, \Lambda^{k(\Phi)}(\Phi)\right), \quad \Lambda^{i}: M \rightarrow \mathbb{R},
$$

and $X(\Phi)=\left(X^{1}(\Phi), \ldots, X^{k(\Phi)}(\Phi)\right), X^{i}: M \rightarrow l^{2}, i=1, \ldots, k(\Phi), k: M \rightarrow \mathbb{N} \cup\{0\}$ such that the complete sets of eigenvalues and eigenvectors of $L_{\Phi}$ can be represented correspondingly as $\bigcup_{x \in \mathbb{Z}^{d}} \Lambda\left(T_{x} \Phi\right)$ and $\bigcup_{x \in \mathbb{Z}^{d}} R_{x} X\left(T_{x} \Phi\right)$.

Let us emphasize that the existence of the function $\Lambda(\Phi)(X(\Phi))$ such that the spectrum of $\mathbf{L}_{\Phi}$ (the set of eigenvectors) can be represented as a union of its values 
along the dynamical system trajectory follows from the mt-property of operator $L_{\Phi}$ and the pure point character of the spectrum. Condition 3) states that such functions can be chosen measurable and almost surely finitely valued. The functions $\Lambda(\Phi)$ and $X(\Phi)$ were introduced by Sinai [4] (which is the reason for the letter S). They were studied in [4] for the almost-Mathieu-type situation. Later they were used in the proof of localization for the $1 D$ discrete Schrödinger operator with two frequency potential [13]. Similar objects were used to study singularcontinuous spectrum [14]. Following the Fröhlich-Spencer construction [15] one can build functions $\Lambda(\omega)$ and $X(\omega)$ satisfying 3 ) for almost every realization $\omega$ in the Anderson localization regime for multi-dimensional Schrödinger operator with random potential.

It seems that generically pure point spectrum of mt-operators satisfies S-conditions 2), 3). The only counterexample we know corresponds to the $1 D$ Schrödinger operator with random discrete-valued potential which possesses pure point spectrum but has the singular component in the integrated density of states [16]. It has been already mentioned however that the case of the discontinuous function on the phase space $\mathbf{M}$ needs generally speaking a special approach and the results presumably are principally different from those for the continuous function. Furthermore, the results related to the Maryland model (see [17]) and Kotani theorem [18] concerning the almost periodic Schrödinger operator with discrete valued potential give rise to a natural question: does there exist an operator of the form (1) with essentially discontinuous functions $f$ and absolutely continuous spectrum? Our conjecture is no.

We will show now that quasiperiodic operators (1) with $\mathbf{S}$-spectrum turns under the transforms of $S L(2, \mathbb{Z}) \backslash \mathbf{G}$ into an operator with absolutely continuous spectrum. It seems that the symbol $\mathbf{S}$ in all considerations may be replaced by "pure point."

To avoid some technical difficulties let us restrict ourselves to the case of finite matrices $B$. Denote by $\mathfrak{A}$ the class of $\mathbf{H}_{B} \in \Sigma$ with finite $B$. Every $\mathbf{H}_{B} \in \mathfrak{A}$ is self adjoint.

Theorem 3. The decomposition of $\mathfrak{U}$ into orbits $\mathbf{O}$ of $S L(2, \mathbb{Z})$-action satisfies the following properties:

1) $\mathbf{N}_{B}$ is constant along the orbit $\mathbf{O}$.

2) There is at most one class of operators with $\mathbf{S}$-spectrum in the every factor-set $\mathbf{O} / \mathbf{G}$; if it exists then all other classes in $\mathbf{O} / \mathbf{G}$ correspond to operators with absolutely continuous spectrum.

Proof. Statement 1) is a corollary of Theorem 2. Let us give the proof of 2).

Define the unitary action on $\mathbb{L}_{2}\left(S^{1} \times \mathbb{Z}\right)$ for some transforms belonging to $S L(2, \mathbb{Q})$.

Set

$$
\begin{aligned}
& \mathbf{U}\left(\begin{array}{ll}
1 & r \\
0 & 1
\end{array}\right): \mathbb{L}_{2}\left(S^{1} \times \mathbb{Z}\right) \rightarrow \mathbb{L}_{2}\left(S_{q}^{1} \times \mathbb{Z}\right) \text { for } r=p / q . \text { Here } S_{q}^{1}=\mathbb{R}(\bmod 2 \pi q), S^{1}=S_{1}^{1} . \\
& \text { Let } \mathfrak{H}=\left\{C=\left(\begin{array}{ll}
a & c \\
b & d
\end{array}\right) \in S L(2, \mathbb{Q}), a, b \in \mathbb{Z}\right\} .
\end{aligned}
$$

Given $C \in \mathfrak{S}$ there exist (not unique) $r_{1}, r_{2} \in \mathbb{Q}, C^{1} \in S L(2, \mathbb{Z})$ :

$$
C=\left(\begin{array}{cc}
1 & r_{1} \\
0 & 1
\end{array}\right) C^{1}\left(\begin{array}{cc}
1 & r_{2} \\
0 & 1
\end{array}\right)
$$


Set

$$
\mathbf{U}_{C}=\mathbf{U}\left(\begin{array}{cc}
1 & r_{1} \\
0 & 1
\end{array}\right) \mathbf{U}_{C^{1}} \mathbf{U}^{1}\left(\begin{array}{cc}
1 & r_{2} \\
0 & 1
\end{array}\right)
$$

It can be shown easily that the definition of $\mathbf{U}_{C}$ is independent of the concrete representation (17) of $C$. Let $q=q(C)$ be the common denominator of $c$ and $d$. Define $\widetilde{C} B(i, j)=B\left(C^{-1}(i, j)\right), i \in \mathbb{Z} / q, j \in \mathbb{Z}, r=p / q, B \in \Sigma$. Here $B(i, j)$ is assumed to be zero for $i \notin \mathbb{Z}$ or $j \notin \mathbb{Z}$.

Lemma 1. Given $C \in \mathfrak{H}, B \in \Sigma$ then the operator family $\mathbf{H}_{\tilde{C} B}^{\Phi}, \Phi \in S_{q(C)}^{1}$ of form (1) is well defined. The functions $f_{j}$ in the related formula (1) belong to $C\left(S_{q}^{1}\right),-\infty<j<\infty$. The operators $\mathbf{H}_{B}^{\Phi}, \Phi \in S^{1}$ and $\mathbf{H}_{\tilde{C}}^{\tilde{\Phi}}, \widetilde{\Phi} \in S_{q}^{1}$ are conjugated through the unitary operator $\mathbf{U}_{C}$. Besides that $\mathbf{N}_{\hat{C} B}(E)=\mathbf{N}_{B}(E)$.

Proof. Define $\mathbf{H}_{\hat{C} B}$ through formula (1) with functions $\widetilde{f}_{j}(x)$ of the form

$$
\sum_{k=-\infty}^{\infty}(\tilde{C} B)(k, j) \exp (i k(x-j \alpha / 2)), \quad x \in S_{q}^{1}, k \in \frac{1}{q} \mathbb{Z} .
$$

It follows that $\tilde{f}_{j}(x)$ is equal to

$$
\sum_{k \in \frac{1}{q} \mathbb{Z}} B\left(C^{-1}(k, j)\right) \exp (i k(x-j \alpha / 2)) .
$$

It remains to repeat the arguments of Proposition 2. To prove the equality of integrated densities of states it suffices to consider random walk on $\mathbb{Z}_{q}^{2}$ which is $\{(m / q, n), m, n \in \mathbb{Z}\}$, and to repeat the proof of Theorem 2 .

Note that $\mathbf{U}_{A C}=\mathbf{U}_{A} \mathbf{U}_{C}$ for $A, C, A C \in \mathfrak{H}$. Every automorphism

$$
\left(\begin{array}{ll}
a & b \\
c & d
\end{array}\right) \in S L(2, \mathbb{Z})
$$

can be represented in the form

$$
\left(\begin{array}{ll}
a & b \\
c & d
\end{array}\right)=\left(\begin{array}{cc}
1 & a c \\
0 & 1
\end{array}\right)\left(\begin{array}{cc}
0 & -1 \\
1 & 0
\end{array}\right)\left(\begin{array}{cc}
c & 0 \\
0 & 1 / c
\end{array}\right)\left(\begin{array}{cc}
1 & d / c \\
0 & 1
\end{array}\right)
$$

As far as operators $\mathbf{U}\left(\begin{array}{ll}1 & r \\ 0 & 1\end{array}\right)$ are unitary in $l^{2}$ for every fixed $\Phi \in S^{1}$ (they don't "mix" $\Phi)$ it suffices to study the action of $\mathbf{U}\left(\begin{array}{cc}0 & -1 \\ 1 & 0\end{array}\right)\left(\begin{array}{cc}c & 0 \\ 0 & 1 / c\end{array}\right)$.

Denote the matrix $\left(\begin{array}{cc}c & 0 \\ 0 & 1 / c\end{array}\right)$ by $D_{c}$. It is straightforward to check that the operator $\mathbf{U}_{D_{C}}$ acts as follows:

$$
\left(\mathbf{U}_{D_{C}} \varphi\right)(\beta, n)=\varphi(\beta / c+\alpha\{n / c\},[n / c]), \quad c \in \mathbb{Z} .
$$

Lemma 2. 1) If the operator $\mathbf{H}_{B} \in \mathfrak{U}$ has pure point spectrum then the spectrum of $\mathbf{H}_{\hat{D}_{c} B}=\mathbf{U}_{D_{c}} \mathbf{H}_{B} \mathbf{U}_{D_{c}}^{-1}$ is also pure point.

2) The $\mathbf{S}$-property is also preserved under the $\mathbf{U}_{D_{c}}$ conjugation.

Proof. Set $X_{k}^{r}(\beta, m)=\Psi_{k}\left(\frac{\beta+\alpha r}{c}, \frac{m-r}{c}\right) \delta(r, m(\bmod c)), 0 \leqq r<c$, for an eigenfunction $\Psi_{K}(\beta)$ of the operator $\mathbf{H}_{B, \alpha}^{\beta}$ with the corresponding eigenvalue $E_{k}(\beta)$. Then

$$
\left\|X_{k}^{r}(\beta)\right\|=\sum_{n \in \mathbb{Z}}\left|X_{k}^{r}(\beta, n)\right|^{2}=\left\|\Psi_{k}\left(\frac{\beta+\alpha r}{c}\right)\right\|<\infty
$$


for any $\beta \in S_{C}^{1}$. It is obvious that $\mathbf{H}_{D_{c} B, \alpha}^{\beta} X_{k}^{r}(\beta)=E_{k}\left(\frac{\beta+\alpha_{r}}{c}\right) X_{k}^{r}(\beta)$. The completeness of the eigenfunction system $\left\{X_{k}^{r}(\beta)\right\}_{k=-\infty}^{\infty}, 0 \leqq r<c$, follows immediately from the completeness of the system $\left\{\Psi_{k}^{r}(\beta)\right\}_{k=-\infty}^{\infty}$ for every $0 \leqq r<c$.

Statement 1 of the S-property is already proven. Statement 2 follows from Lemma 1. Go on to the proof of Statement 3.

Let $\Lambda(\beta)$ and $X(\beta), \beta \in S^{1}$, be measurable functions related to the operator $\mathbf{H}_{B}$. Set

$$
\tilde{\Lambda}(\beta)=\Lambda(\beta / c), \tilde{X}(\beta, m)=X(\beta / c, m / c) \delta(0, m(\bmod c)), \quad \beta \in S_{c}^{1} .
$$

It follows from Statement 1 of the lemma that the spectrum of $\mathbf{H}_{\hat{D}_{c} B, \alpha}^{\beta}$ can be represented as $\bigcup_{k \in \mathbb{Z}} \bigcup_{r=0}^{c-1} E_{k}\left(\frac{\beta+\alpha r}{c}\right)$, where $\left\{E_{k}(\beta)\right\}_{k \in \mathbb{Z}}$ is the spectrum of $\mathbf{H}_{B, \alpha}^{\beta}$. Hence the spectrum of $\mathbf{H}_{\hat{D}_{c} B, \alpha}^{\beta}$ can be written as follows:

$$
\bigcup_{r=0}^{c-1} \bigcup_{k \in \mathbb{Z}} \Lambda\left(\frac{\beta+\alpha r}{c}+k \alpha\right)=\bigcup_{k \in \mathbb{Z}} \Lambda\left(\frac{\beta+\alpha k}{c}\right)=\bigcup_{k \in \mathbb{Z}} \tilde{\Lambda}(\beta+k \alpha) \text {. }
$$

The set of eigenfunctions of $\mathbf{H}_{\hat{D}_{c} \boldsymbol{B}, \alpha}^{\beta}$ can be represented in an analogous way:

$$
\begin{aligned}
& \bigcup_{k \in \mathbb{Z}} \bigcup_{r=0}^{c-1} X_{k}^{r}(\beta)=\bigcup_{k \in \mathbb{Z}} \bigcup_{r=0}^{c-1} \Psi_{k}\left(\frac{\beta+\alpha r}{c}, \frac{m-r}{c}\right) \delta(r, m(\bmod c)) \\
& =\bigcup_{r=0}^{c-1} \bigcup_{k \in \mathbb{Z}} X\left(\frac{\beta+\alpha r}{c}+k \alpha, \frac{m-r}{c}-k\right) \delta(r, m(\bmod c)) \\
& =\bigcup_{k \in \mathbb{Z}} X\left(\frac{\beta+\alpha k}{c}, \frac{m-k}{c}\right) \delta((k-m)(\bmod c), 0)=\bigcup_{k \in \mathbb{Z}} \tilde{X}(\beta+k \alpha, m-k) .
\end{aligned}
$$

Denote by $\mathbf{I}$ the symplectic matrix $\left(\begin{array}{rr}0 & 1 \\ -1 & 0\end{array}\right)$.

Lemma 3. Assume $\mathbf{H}_{B, \alpha}^{\beta} \in \mathfrak{A}$ possess $\mathbf{S}$-spectrum. Then the spectral measure $\mu_{B}^{\beta}(E)$ of $\mathbf{H}_{I B, \alpha}^{\beta}$ is purely absolutely-continuous for almost all $\beta \in[0,2 \pi]$, the generalized eigenfunctions being spectrally almost surely quasiperiodic in the $l^{2}$ sense.

Proof. Using Statement 3 of the S-property write the sets of eigenvalues and eigenfucntions of $\mathbf{H}_{B, \alpha}^{\beta}$ in the form $\bigcup_{m \in \mathbb{Z}} \Lambda(\beta+m \alpha)$ and $\bigcup_{m \in \mathbb{Z}} X(\beta+m \alpha, n-m)$ for measurable functions $\Lambda(\beta)$ and $X(\beta)$ on $S^{1}$. Mimicking Delyon-Chulaevskii's argument (which uses effectively up to this moment only conditions 1, 3 from the $\mathbf{S}$-definition rather than almost-Mathieu specificity) we obtain for the dense set of $\Psi$ in $\mathbb{L}_{2}\left(S^{1} \times \mathbb{Z}\right)$,

$$
\Psi(\beta, n)=\int_{0}^{2 \pi} d \Phi G(\Phi, \beta, n) \sum_{q \in \mathbb{Z}} G^{*}(\Phi, \beta, q) \Psi(\beta, q)
$$

where

$$
G(\Phi, \beta, n)=\sum_{m \in \mathbb{Z}} \exp (i \Phi n+i m(\beta+n \alpha)) X(\Phi, m)
$$

is a multi-(but a.e. finitely)valued function. Relation (19) assumes the summation over all branches of multivalued function $G(\Phi, \beta, n)$ which is

$$
\left(G^{1}(\Phi, \beta, n), \ldots, G^{k(\Phi)}(\Phi, \beta, n)\right)
$$


in correspondence with the branches $\left(\Lambda^{1}(\Phi), \ldots, \Lambda^{k(\Phi)}(\Phi)\right)$ of function $\Lambda(\Phi)$. One can easily check that for a.e. $\beta$ the function $G^{i}(\Phi, \beta, n)$ is a generalized eigenfunction of $\mathbf{H}_{\hat{I} B, \alpha}^{\beta}$ with eigenvalue $\Lambda^{i}(\Phi)$. Relation (19) yields that the type of the spectral measure $\mu_{B}^{\beta}$ of $\mathbf{H}_{I B, \alpha}^{\beta}$ is measure $\mu$, which is $\mu(A)=\int_{0}^{2 \pi} d \Phi \int_{k=1}^{k(\Phi)} \chi\left(\Lambda^{k}(\Phi), A\right)$ for the Borel subsets $A \subset \mathbb{R}$ ( $\chi$ is the characteristic function). Thus $\mu_{B}^{\beta}$ do not depend on $\beta$ and taking into account (3) one obtains the spectral type of $\mathbf{H}_{B}^{\beta}$ to be equal to $d \mathbf{N}_{B}(\lambda)$. The S-condition 2 gives immediately the statement of the lemma.

The assertion 2 of Theorem 3 follows from (18) together with Lemmas 3, 2 because of unitarity in $l^{2}$ of operators $\mathrm{U}\left(\begin{array}{ll}1 & r \\ 0 & 1\end{array}\right)$. Q.E.D.

Let us describe the spectral properties of $S L(2, \mathbb{Z})$-orbit of almost-Mathieu type operators as an example of application of Theorem 3.

Let $\mathbf{H}_{\boldsymbol{B}}$ be the operator of the form (2) with cosine replaced by $\varphi: S^{1} \rightarrow \mathbb{R}$ - the trigonometrical polynomial having exactly one nondegenerate maximum and minimum (see [4]). The last condition can be expressed in terms of algebraic inequalities for the coefficients $b_{j, 0}$ of $\varphi(x)$ "Fourier expansion," setting actually some decreasing property.

Theorem 4. For sufficiently small $\lambda$, typical $\alpha \in S^{1}$ and $A \in S L(2, \mathbb{Z}) / G$ the spectrum of $\mathbf{H}_{\hat{A} \boldsymbol{B}, \alpha}$ is purely absolutely continuous and is a Cantor set of positive Lebesgue measure.

Theorem 4 is an obvious consequence of [4] and Theorem 3.

Let us describe an explicit form of the almost-Mathieu $S L(2, \mathbb{Z})$-orbit.

Let $\mathbf{H}_{B, \alpha}^{\Phi}$ be an almost-Mathieu operator (2). For $A=\left(\begin{array}{ll}m & p \\ n & q\end{array}\right) \in S L(2, \mathbb{Z})$ operator $\mathbf{H}_{\tilde{A} B, \alpha}^{\Phi}$ has the form

$$
\begin{aligned}
\left(\mathbf{H}_{m n p q, \alpha}^{\Phi} \Psi\right)_{l}= & \lambda \Psi_{l+n} \exp \{\operatorname{im}(\alpha(l+n / 2)+\Phi)\}+\Psi_{l+q} \exp \{i p(\alpha(l+q / 2)+\Phi)\} \\
& +\lambda \Psi_{l-n} \exp \{-i m(\alpha(l-n / 2)+\Phi)\}+\Psi_{l-q} \exp \{-i p(\alpha(l-q / 2)+\Phi)\} .
\end{aligned}
$$

Corollary. 1) Under the condition of Theorem 4 the spectrum of $\mathbf{H}_{m n p q, \alpha}^{\Phi}$ is purely absolutely continuous and is a cantor set of positive measure.

2) For a dense $G_{\delta}$ of pairs $(\alpha, \lambda)$ the spectrum is a nowhere dense set.

Assertion 2 follows from the results of [19] and Theorem 2.

Let us emphasize that Theorem 3 contradicts the belief that pure point and absolutely continuous spectra are dual to each other under the discrete Fourier transform. Indeed the operator $\mathbf{U}_{I}$ transforms the pure point spectrum into an absolutely continuous one, though the latter is preserved (generically) under the $\mathbf{U}_{I}$ action.

Example. Consider the family $\tilde{\mathbf{H}}_{\lambda, \alpha}^{\Phi}$ of the form

$$
\begin{aligned}
\left(\tilde{\mathbf{H}}_{\lambda, \alpha}^{\Phi} \Psi\right)_{l}= & \{\lambda \exp \{i(\alpha(l+1 / 2)+\Phi)\}+\exp \{-i(\alpha(l+1 / 2)+\Phi)\}\} \Psi_{l+1} \\
& +\{\lambda \exp \{-i(\alpha(l-1 / 2)+\Phi)\}+\exp \{i(\alpha(l-1 / 2)+\Phi)\}\} \Psi_{l-1}
\end{aligned}
$$

This family is related to the model with pure diagonal hoppings. The "Fourier transform" $\mathbf{U}_{I}$ turns the operator $\tilde{\mathbf{H}}_{\lambda, \alpha}$ into $\tilde{\mathbf{H}}_{\lambda,-\alpha}$ though assuming $\lambda$ or $1 / \lambda$ sufficiently small and $\Phi \in \mathbb{S}^{1}$ being typical the spectrum of $\tilde{\mathbf{H}}_{\lambda, \alpha}$ is pure absolutely continuous. It follows from the fact that $\tilde{\mathbf{H}}_{\lambda, \alpha}$ belongs to the $\operatorname{SL}(2, \mathbb{Z})$ orbit of the almost-Mathieu operator $\mathbf{H}_{\lambda, 2 \alpha}$ of the form (2). 


\section{The Next-Nearest-Neighbor Model}

In this section we will study spectral properties "up to" the singular continuous spectrum of the certain family of the operators $\mathbf{H}_{B}$, i.e. we will prove the absence of absolutely-continuous (point) components in the spectra depending on the parameters given. We will use the equalities on $\mathbf{N}(E)$ together with the connection between the Lyapunov exponent $\gamma(E)$ and the measure $d \mathbf{N}(E)$ in the same way as it was done in $[8,7]$. Here we will deal only with the second order operators $\mathbf{H}$ rather than with the general case. Under such a restriction the Lyapunov exponent $\gamma(E)$ is defined as

$$
\gamma(E)=\sup _{\beta \in[0,2 \pi]} \lim _{n \rightarrow \infty} n^{-1} \ln \left|u_{n}^{2}+u_{n-1}^{2}\right|^{1 / 2}
$$

for the solution $u_{n}$ of the Cauchy problem

$$
\mathbf{H} u=E u
$$

with the initial data

$$
u_{-1}=\cos \beta, \quad u_{0}=\sin \beta .
$$

The limit in (21) exists almost surely and is nonnegative for every mt-operator due to the multiplicative ergodic theorem [20]. The connection between $\gamma(E)$ and $\mathbf{d N}(E)$ in the Schrödinger case is called the Thouless formula. We need a slightly different version of it valid for all $\mathrm{mt}$ second order operators of the form

$$
(\mathbf{H} \Psi)_{n}=a_{n} \Psi_{n+1}+a_{n-1} \Psi_{n-1}+q_{n} \Psi_{n} .
$$

For bounded stationary processes $a_{n}, q_{n}$ and a.e. $E \in \mathbb{R}$ it follows that

$$
\gamma(E)=-\left\langle\ln \left|a_{n}\right|\right\rangle+\int_{-\infty}^{\infty} \ln \left|E-E^{\prime}\right| d \mathbf{N}\left(E^{\prime}\right),
$$

where $\langle$,$\rangle denotes the mathematical expectation.$

Consider the operator family $\mathbf{H}_{\lambda_{1-4}}$ related to the next-nearest-neighbor walk in $\mathbb{Z}^{2}$. The corresponding diagram is

Fig. 2

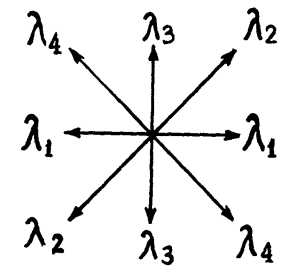

and the family acts in $l^{2}$ through the formula

$$
\begin{aligned}
& \left(\mathbf{H}_{\lambda_{1-4}, \alpha}^{\Phi} \Psi\right)_{l}=2 \lambda_{1} \cos (\alpha l+\Phi) \Psi_{l} \\
& \quad+\left\{\lambda_{3}+\lambda_{2} \exp \{i(\alpha(l+1 / 2)+\Phi)\}+\lambda_{4} \exp \{-i(\alpha(l+1 / 2)+\Phi))\right\} \Psi_{l+1} \\
& \quad+\left\{\lambda_{3}+\lambda_{2} \exp \{-i(\alpha(l-1 / 2)+\Phi)\}+\lambda_{4} \exp \{i(\alpha(l-1 / 2)+\Phi)\}\right\} \Psi_{l-1} .
\end{aligned}
$$

Set $\lambda=\max \left(\lambda_{1}, \lambda_{3}, \lambda_{2}+\lambda_{4}\right)$.

Theorem 5. For any irrational $\alpha$ and a.e. $\Phi \in S^{1}$,

1) For $\lambda=\lambda_{1}$ being a strict maximum there is no absolutely continuous component in 
the spectrum of $\mathbf{H}_{\lambda_{1-4}, \alpha}^{\Phi}$. The Lyapunov exponents (if any) on the spectrum are given by the formulas:

$$
\begin{array}{ll}
\gamma(E)=2 \pi \ln \frac{\lambda_{1}+\sqrt{\lambda_{1}^{2}-4 \lambda_{2} \lambda_{4}}}{2 \lambda_{2}}, & \lambda_{2}>\lambda_{4}, \lambda_{3}<\lambda_{4}+\lambda_{2} ; \\
\gamma(E)=2 \pi \ln \frac{\lambda_{1}+\sqrt{\lambda_{1}^{2}-4 \lambda_{2} \lambda_{4}}}{2 \lambda_{4}}, & \lambda_{2}<\lambda_{4}, \lambda_{3}<\lambda_{4}+\lambda_{2} ; \\
\gamma(E)=2 \pi \ln \frac{\lambda_{1}+\sqrt{\lambda_{1}^{2}-4 \lambda_{2} \lambda_{4}}}{\lambda_{3}+\sqrt{\lambda_{3}^{2}-4 \lambda_{2} \lambda_{4}}}, & \lambda_{3}>\lambda_{4}+\lambda_{2} .
\end{array}
$$

2) For $\lambda=\lambda_{3}$ being a strict maximum there is no pure point component in the spectrum of $\mathbf{H}_{\lambda_{1-4}, \alpha}^{\Phi}$. The Lyapunov exponents (if any) are equal to zero.

3) For $\lambda=\lambda_{2}+\lambda_{4}$ being a strict maximum the Lyapunov exponents (if any) on the spectrum are equal to zero.

Remark. 1. Setting $\lambda_{2}=\lambda_{4}=0$ turns $\mathbf{H}_{\lambda_{1-4}, \alpha}^{\Phi}$ into the almost-Mathieu operator for which the results of Theorem 5 were proved in [7-9].

2. The case when $\lambda$ is not a strict maximum has codimension 1 and seems to correspond to the purely singular continuous spectrum (see below).

Proof. According to (24) for a.e. $E \in \mathbb{R}$ the Lyapunov exponent $\gamma\left(\lambda_{1-4} ; E\right)$ of operator $\mathbf{H}_{\lambda_{1-4, \alpha}}^{\Phi}$ is equal to

$$
\gamma\left(\lambda_{1-4} ; E\right)=-\int_{0}^{2 \pi} \ln \left|\lambda_{3}+\lambda_{2} e^{i x}+\lambda_{4} e^{-i x}\right| d x+\int_{-\infty}^{\infty} \ln \left|E-E^{\prime}\right| d \mathbf{N}_{\lambda_{1-4}}\left(E^{\prime}\right) .
$$

Denote by $\sigma$ the transposition $(1,3)$. According to Theorem $2 \mathbf{N}_{\lambda_{1-4}}(E)$ $=\mathbf{N}_{\lambda_{\sigma(1-4)}}(E)$, thus (25) can be rewritten as

$$
\begin{aligned}
\gamma\left(\lambda_{1-4} ; E\right) & =-\int_{0}^{2 \pi} \ln \left|\lambda_{3}+\lambda_{2} e^{i x}+\lambda_{4} e^{-i x}\right| d x+\int_{-\infty}^{\infty} \ln \left|E-E^{\prime}\right| d \mathbf{N}_{\lambda_{\sigma(1-4)}}\left(E^{\prime}\right) \\
& =\gamma\left(\lambda_{\sigma(1-4)} ; E\right)+2 \pi \int_{0}^{2 \pi} \ln \left|\frac{\lambda_{1}+\lambda_{2} e^{i x}+\lambda_{4} e^{-i x}}{\lambda_{3}+\lambda_{2} e^{i x}+\lambda_{4} e^{-i x}}\right| d x .
\end{aligned}
$$

Denote $2 \pi \int_{0}^{2 \pi} \ln \left|\frac{\lambda_{1}+\lambda_{2} e^{i x}+\lambda_{4} e^{-i x}}{\lambda_{3}+\lambda_{2} e^{i x}+\lambda_{4} e^{-i x}}\right| d x$ by $L\left(\lambda_{1-4}\right)$.

Since $\gamma(E)$ cannot be negative the positivity of $L\left(\lambda_{1-4}\right)$ implies the positivity of $\gamma\left(\lambda_{1-4} ; E\right)$ for a.e. $E$.

The straightforward calculation shows that

$$
\begin{array}{ll}
L\left(\lambda_{1-4}\right)=2 \pi \ln \frac{\lambda_{1}+\sqrt{\lambda_{1}^{2}-4 \lambda_{2} \lambda_{4}}}{2 \lambda_{2}}, & \lambda_{2}>\lambda_{4}, \lambda_{3}<\lambda_{4}+\lambda_{2}, \lambda_{1}>\lambda_{4}+\lambda_{2}, \\
L\left(\lambda_{1-4}\right)=2 \pi \ln \frac{\lambda_{1}+\sqrt{\lambda_{1}^{2}-4 \lambda_{2} \lambda_{4}}}{2 \lambda_{4}}, & \lambda_{2}<\lambda_{4}, \lambda_{3}<\lambda_{4}+\lambda_{2}, \lambda_{1}>\lambda_{4}+\lambda_{2}, \\
L\left(\lambda_{1-4}\right)=2 \pi \ln \frac{\lambda_{1}+\sqrt{\lambda_{1}^{2}-4 \lambda_{2} \lambda_{4}}}{\lambda_{3}+\sqrt{\lambda_{3}^{2}-4 \lambda_{2} \lambda_{4}}}, & \lambda_{3}>\lambda_{4}+\lambda_{2}, \lambda_{1}>\lambda_{4}+\lambda_{2},
\end{array}
$$




$$
\begin{array}{ll}
L\left(\lambda_{1-4}\right)=-2 \pi \ln \frac{\lambda_{3}+\sqrt{\lambda_{3}^{2}-4 \lambda_{2} \lambda_{4}}}{2 \lambda_{2}}, & \lambda_{2}>\lambda_{4}, \lambda_{3}>\lambda_{4}+\lambda_{2}, \lambda_{1}<\lambda_{4}+\lambda_{2}, \\
L\left(\lambda_{1-4}\right)=-2 \pi \ln \frac{\lambda_{3}+\sqrt{\lambda_{3}^{2}-4 \lambda_{2} \lambda_{4}}}{2 \lambda_{4}}, & \lambda_{2}<\lambda_{4}, \lambda_{3}>\lambda_{4}+\lambda_{2}, \lambda_{1}<\lambda_{4}+\lambda_{2}, \\
L\left(\lambda_{1-4}\right)=0, \quad \lambda_{1}, \lambda_{3}<\lambda_{4}+\lambda_{2} . &
\end{array}
$$

It remains to use the argument of [21] to obtain assertion 1. Assertions 2, 3 can be proved by the argument of [9] which being repeated in our situation shows that $\gamma\left(\lambda_{1-4} ; E\right)>0$ implies $\gamma\left(\lambda_{\sigma(1-4)} ; E\right)=0$. In fact

$$
\mathbf{H}_{\lambda_{1-4}, \alpha}^{\Phi}=\mathbf{U}_{I} \mathbf{H}_{\lambda_{\sigma(1-4), \alpha}}^{\Phi} \mathbf{U}_{I}^{-1}
$$

and it remains to notice that Delyon's argument exploits only the properties of the Fourier transform. Q.E.D.

Let us restrict ourselves to the case $\lambda_{2}=\lambda_{4}$. Setting $\lambda_{1}=1$ we obtain the following diagram in the plane $\lambda_{2}, \lambda_{3}$

Fig. 3

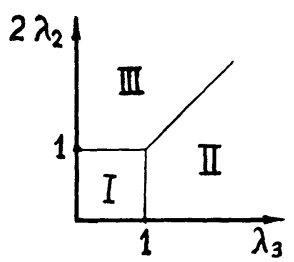

Domain I corresponds to the absence of the purely continuous part in the spectrum, Domain II to the absence of the pure point component, and Domain III cannot be studied by our methods but it seems to correspond to the singularcontinuous spectrum.

The latter model was considered in [22] where the expressions for the Lyapunov exponents were announded in the domain $\lambda_{3}>1$ and the numerical computations for the $\left\{\operatorname{III} \cap\left\{\lambda_{3}>1\right\}\right\}$ were fulfilled. The results of computations give rise in regard to the spectrum of operators corresponding to III as singularcontinuous.

It can be stated more for the case: $\lambda_{2}=0$, which is reverse in a sense to the previous one. It turns out that operator $\mathbf{H}_{\lambda_{1-4}}$ in this case corresponds to the random walk on the triangular lattice.

Indeed consider the lattice $\widetilde{\mathbb{Z}}^{2}$ with the cell of the form of a parallelogram having sides $a, b$ and the angle $\beta \in[0, \pi]$. We regard the area of the unit cell $a b \sin \beta$ as being equal to 1 . The argument used to prove Theorem 1 repeated with appropriate changes shows that under some "rationality" conditions the corresponding partition function $\widetilde{\Xi}_{B, \alpha ; t}$ (defined with respect to $\widetilde{\mathbb{Z}}^{2}$ in the same way as $\Xi_{B, \alpha ; t}$ with respect to $\mathbb{Z}^{2}$; see 2) is independent of $a, b, \beta$ and is equal to $\Xi_{B, \alpha ; t}$. The "rationality" condition mentioned is that $b^{2} \sin 2 \beta \in \mathbb{Q}$. Thus all "rational" in the above sense lattices are equivalent.

The triangular walk can be regarded as a walk on the lattice $\tilde{\mathbb{Z}}^{2}$ with parameters $a=b=\sqrt{\frac{2}{\sqrt{3}}}, \sin \beta=\frac{\sqrt{3}}{2}$ (which implies $b^{2} \sin 2 \beta=1$ ). Hence the corresponding 
diagram is

Fig. 4

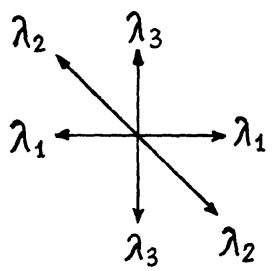

and the corresponding operator is $\mathbf{H}_{\lambda_{1} \lambda_{2} \lambda_{3} 0}:=\mathbf{H}_{\lambda_{1} \lambda_{2} \lambda_{3}}$.

Theorem 6 (the triality). Given transposition $\sigma=\left(\sigma_{1}, \sigma_{2}, \sigma_{3}\right)$ the following equality holds:

$$
\mathbf{N}_{\lambda_{1-3}}(E)=\mathbf{N}_{\lambda_{\sigma_{1}-\sigma_{3}}}(E) \text {. }
$$

Proof. The partition function $\widetilde{\mathbb{\Xi}}_{B, \alpha ; t}$ corresponding to the lattice $\tilde{\mathbb{Z}}^{2}$ with parameters $a, b, \beta$ defined above is invariant with respect either to the $\pi / 3$-rotations as to reflections in the axes $\{x=0\},\{y=0\},\{y=-x\}$ which together with (3) gives immediately the desired equality.

Theorem 7. For irrational $\alpha$, almost every $\Phi \in \mathbb{S}^{1}$ and $\lambda_{2}>\lambda_{1}, \lambda_{2}>\lambda_{3}$ there are no pure point component in the spectrum of $\mathbf{H}_{\lambda_{1-3, \alpha}}^{\Phi}$.

Remark. All the other domains in the space $\left(\lambda_{1}, \lambda_{2}, \lambda_{3}\right)$ are already regarded in Theorem 5.

Proof. It follows from Theorem 5 that for $\lambda_{1}>\lambda_{2}, \lambda_{1}>\lambda_{3}$ the Lyapunov exponent $\gamma\left(\lambda_{1-3} ; E\right)$ is strictly positive for $E$ belonging to the spectrum. It remains to note that $\mathbf{H}_{\lambda_{2} \lambda_{1} \lambda_{3}, \alpha}^{\Phi}=\mathbf{H}_{\hat{J} B,-\alpha}^{-\Phi}$ for $\mathbf{J}=\left(\begin{array}{rr}1 & -1 \\ 0 & 1\end{array}\right) \mathbf{I}, \mathbf{H}_{B}=\mathbf{H}_{\lambda_{1-3}}$, and to repeat the reasoning of Theorem 5. Q.E.D.

Acknowledgements. We would like to express our profound gratitude to Ya. G. Sinai for his encouragement and fruitful discussions and to E. I. Dinaburg for reading the manuscript and making many useful remarks.

\section{References}

1. Harper, P.G.: Single band motion of conduction electrons in a uniform magnetic field. Proc. Phys. Soc. Lond. A 68, 874-892 (1955)

2. Dinaburg, E.I., Sinai, Ya.G.: The one-dimensional Schrödinger equation with a quasiperiodic potential. Funct. Anal. Appl. 9, 279-289 (1975)

3. Johnson, R.A., Moser, J.: The rotation number for almost periodic potentials. Commun. Math. Phys. 84, 403-438 (1982)

4. Sinai, Ya.G.: Anderson localization for one-dimensional Schrödinger operator with quasiperiodic potential. J. Stat. Phys. 46, 861-909 (1987)

5. Fröhlich, J., Spencer, T., Wittwer, P.: Localization for a class of one-dimensional quasiperiodic Schrödinger operators. Commun. Math. Phys. 132, 5-25 (1990)

6. Aubry, G., Andre, G.: Analyticity breaking and Anderson localization in incommensurate lattices. Ann. Israel Phys. Soc. 3, 133-140 (1980)

7. Avron, J., Simon, B.: Almost periodic Schrödinger operators II. The integrated density of states. Duke Math. J. 50, 369-385 (1983) 
8. Figotin, A.L., Pastur, L.A.: The positivity of Lyapunov exponent and absence of absolutely continuous spectrum for almost Mathieu equation. J. Math. Phys. 25, 774-777 (1984)

9. Delyon, F.: Absence of localization in the almost Mathieu equation. J. Phys. A 20, L21-L23 (1987)

10. Chulaevsky, V., Delyon, F.: Purely absolutely continuous spectrum for almost Mathieu operators. Preprint, Palaiseau 1989

11. Figotin, A.L.: The ergodic properties and the essential selfadjointness of random matrix operators. In: Operators in functional spaces and problems of the theory of functions, Kiev: Naukova Dumka 1987 (Russian), pp. 13-21

12. Pastur, L.A.: Lower bounds of the Lyapunov exponent for some finite-difference equations with quasiperiodic coefficients. Ibid., 3-13

13. Chulaevsky, V.A., Sinai, Ya.G.: Anderson localization for the $1-D$ discrete Schrödinger operator with two-frequency potential. Commun. Math. Phys. 125, 91-112 (1989)

14. Zhitomirskaya, S.Ya.: Singular spectral properties of a discrete Schrödinger operator with quasiperiodic potential, to appear. In: Sinai, Ya.G. (ed.). Adv. Sov. Math., V.3, Dynamical systems and statistical pyhsics (1991)

15. Fröhlich, J., Spencer, T.: Absence of diffusion in the Anderson tight-binding model for large disorder or low energy. Commun. Math. Phys. 88, 151-189 (1983)

16. Carmona, R., Klein, A., Martinelli, F.: Anderson localization for Bernoulli and other singular potentials. Commun. Math. Phys. 108, 41-66 (1987)

17. Simon, B.: Almost periodic Schrödinger operators IV. The Maryland model. Ann. Phys. 159, 157-183 (1985)

18. Kotani, S.: Jacobi matrices with random potentials taking finitely many values. Preprint, Tokyo 1989

19. Bellisard, J., Simon, B.: Cantor spectrum for the almost Mathieu equation. J. Funct. Anal. 48, 408-419 (1982)

20. Osceledec, V.I.: A multiplicative ergodic theorem. Lyapunov exponents for dynamical systems. Trudy Mosk. Mat. Obsc. 19, 679-713 (1968)

21. Pastur, L.A.: Spectral properties of disordered systems in one-body approximation. Commun. Math. Phys. 75, 179-196 (1980)

22. Thouless, D.J.: Bandwidths for a quasiperiodic tight-binding model, Phys. Rev. 28, 4272-4276 (1983)

Communicated by Ya. G. Sinai 